\title{
Measurement of ultra-trace level of intact oxytocin in plasma using SALLE combined with nano-LC-MS
}

\author{
Dan Liu ${ }^{\mathrm{a}, 1}$, Xiaoli Han ${ }^{\mathrm{a}, 1}$, Xinxin Liu ${ }^{\mathrm{a}, 1}$, Mengchun Cheng ${ }^{\mathrm{a}}$, Meixi He ${ }^{\mathrm{b}}$, Gregor Rainer ${ }^{\mathrm{c}}$, \\ Huiyuan Gao ${ }^{\mathrm{b}, *}$, Xiaozhe Zhang ${ }^{\mathrm{a}, *}$ \\ a CAS Key Laboratory of Separation Sciences for Analytical Chemistry, Dalian Institute of Chemical Physics, Chinese Academy of Sciences, 457 Zhongshan \\ Road, Dalian, 116023, China \\ ${ }^{\mathrm{b}}$ Key Laboratory of Structure-Based Drug Design \& Discovery of Ministry of Education, Shenyang Pharmaceutical University, Shenyang, 110016, China \\ ${ }^{\mathrm{c}}$ Department of Medicine, University of Fribourg, Chemin de Musee 5, Fribourg, CH-1700, Switzerland
}

Keywords:

Oxytocin

SALLE

Nano-LC-MS

Plasma

\begin{abstract}
A B S T R A C T
Measurement of peptides such as oxytocin in plasma is a critical challenge in clinical research because of their extreme low concentrations as well as the tremendous interferencing substances co-presented in plasma. In this study, we developed an efficient salt-out assisted liquid-liquid extraction (SALLE) to treat plasma, and then analyzed the samples using nano-LC-MS to quantify intact oxytocin (OT) in human and rat plasmas. Our results showed that the use of SALLE (Isopropanol $/ \mathrm{K}_{2} \mathrm{HPO}_{4}(4 \mathrm{M})$ ) allows efficient removal of various disrupters, including proteins, inorganic salts, and lipids, which helps avoid the risk of blocked capillary columns and matrix effects. Moreover, instant SALLE can reduce the possible binding between OT and proteins, thus allowing high repeatability of OT extraction from the original plasma. This combination of SALLE and nano-LC-MS method provided in the end a $1 \mathrm{pg} / \mathrm{m} \mathrm{L}$ of detection limit. Comparative analysis showed that the concentration of OT in the plasma taken from 12 volunteers ranged from 3 to $214 \mathrm{pg} / \mathrm{m} \mathrm{L}$, about one order less than those in the plasma of rats. Compared to the previously reported LC-MS and immunoassay methods, the combination of SALLE and nano-LC-MS permits reliable measurement of intact OT even in human plasma. Our approach may be an alternative method for quantitative determination of other ultra-trace peptides in plasma, which would help the investigators understand the role of peptides in behaviours and diseases.
\end{abstract}

\section{Introduction}

Oxytocin (OT) is a crucial neuropeptide (Cys-Tyr-Ile-Gln-AsnCys-Pro-Leu-Gly-HN2) that is able to facilitate childbirth and breastfeeding, and may be associated with complex emotions and social behaviours [1-6]. Human plasma is a readily available clinical sample that reflects the status of the body in normal physiological and disease states. OT has been proved to be involved in the regulation of animal social behaviour. Previous study found autistic children group had significantly lower plasma OT levels than the normal children group, despite individual variability and overlapping group distributions [7]. Although the wide dynamic range of oxytocin and immense complexity of plasma are obstacles, oxy-

\footnotetext{
* Corresponding authors.

E-mail addresses: sypugaohy@163.com (H. Gao), zhangxz@dicp.ac.cn (X. Zhang).

1 The authors contributed equally to this work
}

tocin analysis of human plasma is necessary for understanding the role of this peptide in multiple neurophysiological processes and behaviour.

There are several analytical techniques which have been reported for the analysis of oxytocin [8,9]. Commercial enzyme immunoassay (EIA) $[10,11]$ and radioimmunoassay (RIA) $[12,13]$ methods are generally used for the analysis of oxytocin. The sensitivity, indicated as the lower limit of quantification, can reach up to $\mathrm{pg} / \mathrm{m}$ L. However, antibodies commonly do not have enough specificity to discriminate between intact OT and conjugated OT, OT fragments, or OT-like proteins [8]. Such a low specificity inevitably affects the detection accuracy and can lead to contradictory findings.

An alternative to immunoassay method is mass spectrometry (MS)-based methodology. The extract determination of the $\mathrm{m} / \mathrm{z}$ ratio using MS allows ready discrimination of OT from its various analogues. Currently, the few published mass spectrometer methods for measuring OT in plasma are usually coupled with a nano-LC or a 2D-LC to improve detection sensitive [14-17]. In a study using 
2D-LC-MS, Zhang et al. [16] were able to selectively quantify intact OT in the human plasma at the $\mathrm{pg} / \mathrm{mL}$ level. Brandtzaeg et al. [17] reported a nano-LC-MS platform using reduction/alkylation to measure all circulating OT in human plasma. The concentration of the total OT in pooled human plasma was several hundred $\mathrm{pg} / \mathrm{m} \mathrm{L}$. The measurement of the total OT lowers the analytical challenge. However, measurement of the level of intact OT, rather than bound OT, is desired in most cases, because the former might closely reflect the physical or pathological conditions of bodies.

The efficiency in the removal of highly abundant proteins is one of the main technique challenges in nano-LC-MS analysis of ultratrace peptides. For instance, the capillary column can easily lose its performance after just one injection of protein-precipitated (PPT) plasma. Moreover, the presence of highly abundant lipids and salts make it challenging to isolate polar peptides like OT (XLogP -2.6) [18] through commonly used SPE procedures. This becomes apparent when considering that OT is a small nonapepatide at $\mathrm{pg} / \mathrm{mL}$ level. OT containing a disulfide bond that tends to be easily lost because of the co-precipitation with albumin containing multiple disulfide bonds $[19,20]$. We indeed observed that the intact OT content significantly reduced before sample pre-treatment under normal laboratory conditions, leading to varying results.

Salt-out assisted liquid-liquid extraction (SALLE) might be an alternative method for the pretreatment of plasmas, using an water-miscible organic solvents (i.e. acetonitrile and isopropanol) as extractants, which has been proven to be capable of extracting a wide range of polar drugs from biological matrices that are difficult to separate by SPE [21-24]. The principle of SALLE is based on the phase separation of water-miscible organic solvent from aqueous solution in the presence of high concentration of salts. SALLE is an economical method, suitable for routine analysis purposes. The aim of the present study was to develop an SALLE method combined with nano-LC-MS to accurately determine the content of ultra-trace level of endogenous OT in plasma. No derivatizing reagent has been used in the present study in comparison to previously reported. All the parameters affecting the SALLE have been optimized with aid of experimental design. Under optimized conditions, the developed method has been validated and applied for the estimation of oxytocin in human plasmas and rat plasmas.

\section{Experimental}

\subsection{Chemicals and reagents}

Oxytocin (OT) standard ( $>98 \%$ purity) was purchased from GL Biochem Co., Ltd (Shanghai, China). Isotope-labelled Pro [13C $5,15 \mathrm{~N}$ ] oxytocin (IS) (>98\% purity) was purchased from Thermo Fisher Scientific (U.S.A). Chromatographic-grade acetonitrile (AcN) was obtained from Sigma-Aldrich, Co. (St. Louis, Mo, U.S.A). Ethanol (EtOH) and isopropanol (IPA) of chromatographic-grade were purchased from Spectaum Chemical Mfg. Corp. (California, U.S.A). Methanol (MeOH) was obtained from Yuwang Industry Co., Ltd (Shandong, China). Phosphoric acid, formic acid, dichloromethane (DCM), and dipotassium phosphate $\left(\mathrm{K}_{2} \mathrm{HPO}_{4} \cdot 3 \mathrm{H}_{2} \mathrm{O}\right)$ were purchased from the Kermel Chemical Reagent Co., Ltd (Tianjin, China). Deionized water was purified by a Milli-Q system (Millipore, Bedford, MA, U.S.A). All materials were purchased with low binding attributes (tubes, vials, and pipette tips) to minimize peptide adsorption to the tube and vial walls. The commercial human plasma (MK1016100 NHP) was obtained from Manuik Technology Co., Ltd (Chongqing, China). Vacutainer (BD ${ }^{\mathrm{TM}}$ P800) was purchased from Becton, Dickinson and Company (U.S.A).

Stock solutions of OT and IS were prepared separately at $0.5 \mathrm{mg} / \mathrm{m} \mathrm{L}$ by dissolving $5 \mathrm{mg}$ of dry power in $10 \mathrm{~m} \mathrm{L15 \% AcN}$ and further diluted in deionized water to obtained working solutions of $5 \mathrm{ng} / \mathrm{m} \mathrm{L}$ and stored at $4^{\circ}$.

\subsection{Biological samples}

This study, including the collection and use of all human and rat samples, were approved by the ethics committee of Dalian Municipal Central Hospital Dalian, China and Dalian Medical University, Dalian, China, respectively. All volunteers submitted written consent for inclusion in this study cohort. Whole blood of volunteers (non-pregnant individuals) were collected after $\mathrm{O} / \mathrm{N}$ fasting, between 7 and $8 \mathrm{AM}$, and then centrifuged at $825 \mathrm{~g}$ for $10 \mathrm{~min}$. The supernatant (plasma) was sub-packed and stored at $-80^{\circ}$ until used. The male Wistar rats $(150-180 \mathrm{~g})$ were obtained from the Experimental Animal Center of Dalian Medical University. The rats were deeply anesthetized with chloral hydrate (350 mg/mg, i.p.), then the blood samples were collected by abdominal aortic approach in polythene tubes (BD P800). Subsequent processing was the same as human plasma. The commercial human plasma (MK1016100 NHP) was used for optimization of the SALLE method.

\subsection{Sample preparation}

In $10 \mathrm{~m} \mathrm{~L}$ polypropylene vials, aliquots $(\mathrm{n}=3)$ of plasma $(600 \mu \mathrm{L}$ of human or $300 \mu \mathrm{L}$ of rat) was mixed with IS working solution (24 $\mu \mathrm{L}$ for human or $48 \mu \mathrm{L}$ for rat). Subsequently, $5 \%(\mathrm{v} / \mathrm{v}) \mathrm{H}_{3} \mathrm{PO}_{4}$ ( $240 \mu \mathrm{L}$ for human or $120 \mu \mathrm{L}$ for rat) was added and vigorously vortexed for $30 \mathrm{~s}$. Then, $4 \mathrm{~mL} \mathrm{~K} \mathrm{HPO}_{4}$ aqueous solution $(4 \mathrm{M})$ and $4 \mathrm{~mL}$ IPA were added successively to samples and mixed $30 \mathrm{~s}$ after each step with a vortexer. The mixture was centrifuged $10 \mathrm{~min}$ (3000 rpm). The supernatant $(4 \mathrm{~m} \mathrm{~L})$ was transferred to $5 \mathrm{~m} \mathrm{~L}$ vials and dried under a steady stream of nitrogen. Dried sample was further treated with $1 \mathrm{~mL}$ IPA for desalting. After centrifugation, the supernatant was transferred to $1.5 \mathrm{~m} \mathrm{~L}$ vials and dried. The residue was dissolved in $40 \mu \mathrm{L}$ of $15 \%$ acetonitrile with $0.2 \% \mathrm{FA}$ and $10 \mu \mathrm{L}$ $\mathrm{DCM}$, and then centrifuged $40 \mathrm{~min}(15,000 \mathrm{rpm})$. The supernatant $(5 \mu \mathrm{L})$ was injected into nano-LC-MS system for analysis.

\subsection{Nano-liquid chromatography-mass spectrometry}

The trap column was packed in-house with C18 AQ particles ( $5 \mu \mathrm{m}, 100 \AA ̊$, Dr. Maisch GmbH, Ammerbuch-Entringen, Germany) in a PEEK column holder (Upchurch, Oak Harbor, WA, USA). The column length was $3 \mathrm{~cm}(100 \mu \mathrm{m}$ ID). The analytical column $(25 \mathrm{~cm} \times 75 \mu \mathrm{m})$ with $\mathrm{C} 18$ AQ particles $(3 \mu \mathrm{m}, 100 \AA)$ was packed in a Picofrit capillary equipped with an emitter tip of $10 \mu \mathrm{m}$ (New Objective, Woburn, MA, USA).

LTQ-Orbitrap Discovery (Thermo Fisher Scientific, Bremen, Germany) combined with nano-LC (Eksigent Technologies, California, USA) was employed. In the 1D pump, $5 \mu \mathrm{L}$ of the sample was injected to the trap column. Mobile phases A and B were $0.2 \% \mathrm{FA}$ and $80 \% \mathrm{ACN}$ with $0.2 \% \mathrm{FA}$, respectively. Isocratic elution was $2 \%$ mobile phase $B$ at a flow rate of $3 \mu \mathrm{L} / \mathrm{min}$. The elution direction of trap column was positive through a 6 -port valve in order to combine with the analytical column after the samples were enriched for $7 \mathrm{~min}$ in it. In the 2D pump, the sample was flushed from the trap column onto the separation column with mobile phase A comprising $0.2 \%$ FA and mobile phase B $80 \%$ comprising ACN with $0.2 \%$ FA. The gradient program was $0-5 \mathrm{~min}, 5 \% \mathrm{~B} ; 5-12 \mathrm{~min}, 8-30 \% \mathrm{~B}$; 12-42 min, 30-75\% B; 42-50 min, 75-90\%B; 50-55 min, 100\% B at $0.3 \mu \mathrm{L} / \mathrm{min}$. Data acquisition on the LTQ-Orbitrap Elite system consisted of a full Fourier transform mass spectrometry (FTMS) scan event at the mass range of $504-509.4 \mathrm{~m} / \mathrm{z}$ in positive ion mode, using a resolution setting of 60,000 (full width at half-maximum at $\mathrm{m} / \mathrm{z} 400$ ), operating in the data-dependent mode. Data were managed on the Thermo Xcalibur Data Acquisition software (Version B.04.00, 2.2). 
A

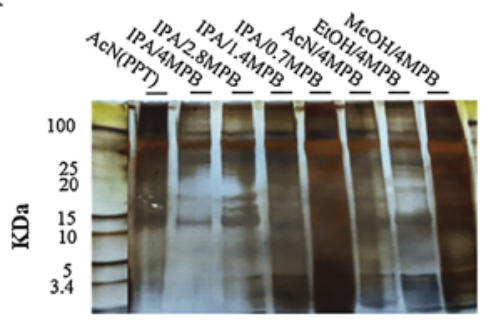

$\mathrm{C}$

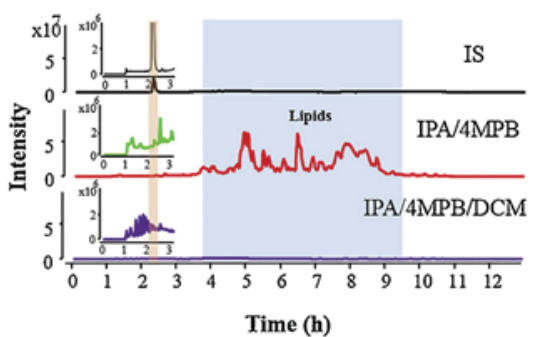

B

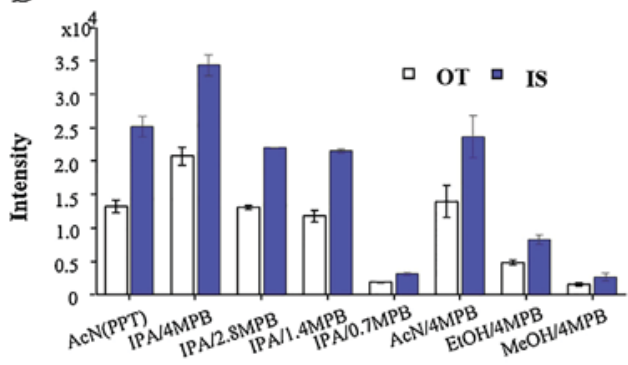

$\mathrm{D}$

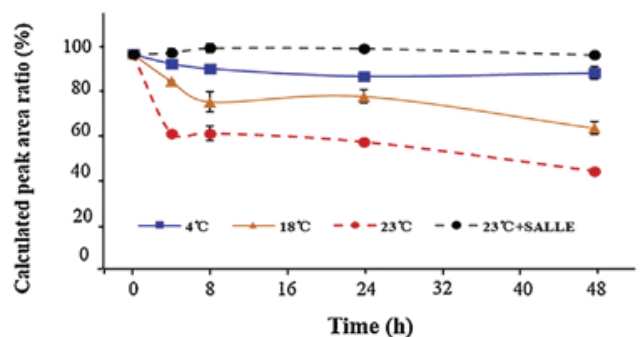

Fig. 1. The electropherogram of plasma extraction obtained with different pretreatment method (A), including salt-out assisted liquid-liquid extraction (SALLE) method with four different extract solvents and four different concentrations of phase-forming salt, and protein precipitation method (PPT), the extraction yields of oxytocin (OT) and IS in spiked commercial human plasma were presented as mean values $\pm S D(n=3)(B)$, the representative total ion chromatograms of plasma extraction and isotope labeled oxytocin (IS) standard solution in positive mode by UPLC-QTOF (C), effect of instant SALLE on intact oxytocin measurement in plasma(D), OT binds to protein under different storage conditions, resulting in varying detection, instant SALLE avoids OT binding to plasma protein, preventing loss of OT during sample preparation. (For interpretation of the references to colour in this figure text, the reader is referred to the web version of this article.)

\subsection{Method validation}

The method of SALLE combined with nano-LC-MS was verified for the limits of quantification, recovery, precision, matrix effect, and calibration factor [25]. The absolute recovery, extract efficiency, and matrix effect were determined using validation samples that were pooled using commercial plasma spiked with OT at three different concentration levels: low $10 \mathrm{pg} / \mathrm{mL}$, middle $60 \mathrm{pg} / \mathrm{mL}$, high $360 \mathrm{pg} / \mathrm{m} \mathrm{L}$, and IS $300 \mathrm{pg} / \mathrm{m} \mathrm{L}$. The rest of the procedure was the same as for the SALLE (see the section above). The intra-day and inter-day precisions were expressed as the percentage relative standard deviation (\%RSD) and evaluated with the above validation samples. Five replicates of each concentration level were used to determine the intra-day precision. This process was repeated three times at three successive days to determine the inter-day precision. The calibration curve was made up of 7 points with $1-1000 \mathrm{pg} / \mathrm{m} \mathrm{L}$ of OT and $200 \mathrm{pg} / \mathrm{mL}$ of IS in a $15 \%$ acetonitrile ( $0.2 \%$ formic acid, $0.05 \%$ human plasma) solution.

\section{Results and discussion}

\subsection{Removal of protein, salt, and lipid}

To find the optimal conditions of SALLE for effectively removing proteins, salts, and lipids, and extraction of intact OT from plasma, a series of water-miscible organic solvents $(\mathrm{MeOH}, \mathrm{EtOH}, \mathrm{IPA}$, and AcN) were investigated. All the selected organic solvents showed excellent solubility for intact OT. $\mathrm{K}_{2} \mathrm{HPO}_{4}$ was selected as phase forming agent, because its weakly alkaline property that favours the protonation of OT (pKa 6.1), making OT less hydrophilic. A pooled commercial human plasma spiked with $30 \mathrm{ng} / \mathrm{m}$ L OT and IS, respectively, was utilized as a model plasma. After evaporation of the extracts, the residue was dissolved in $40 \mu \mathrm{L}$ of $15 \%$ acetonitrile with $0.2 \% \mathrm{FA}$ and $10 \mu \mathrm{L} \mathrm{DCM}$, and then centrifuged $40 \mathrm{~min}$ (15000 rpm). The supernatant was introduced into the chromatographic vial for UPLC-QTOF system analysis.
Fig. 1A shows the electropherogram of the extraction results obtained using different SALLE systems and PPT. SDS-PAGE method was shown in SI. A cleaner electrophoretic map was also obtained using the IPA/ $\mathrm{K}_{2} \mathrm{HPO}_{4}(4 \mathrm{M})$ system, duo to salting-out and organic solvent precipitation, causing the interfering protein to form an intermediate solid layer. Moreover, the role of IPA was to solubilize OT. The high salt concentration causes a further decrease in the water content of the IPA-rich phase, prompting the inorganic salt in the plasma to enter the $\mathrm{K}_{2} \mathrm{HPO}_{4}$-rich phase. The amount of the residual protein of SALLE (IPA $/ \mathrm{K}_{2} \mathrm{HPO}_{4}(4 \mathrm{M})$ ) during the extraction was clearly lower than that of PPT (AcN); as a result, more than $98 \%$ of protein precipitates out [26]. As demonstrated in Fig. 1B, each system shows a specific interaction with OT. Clearly, the SALLE (IPA/ $\mathrm{K}_{2} \mathrm{HPO}_{4}(4 \mathrm{M})$ ) method is much better than other methods to extract intact OT from plasma. This difference was related to the physical location of OT during biphasic extraction. The SALLE $\left(\mathrm{AcN} / \mathrm{K}_{2} \mathrm{HPO}_{4}(4 \mathrm{M})\right)$ method provided a comparable OT yield with AcN precipitation. This shows that the selectivity of organic solvent for OT extraction played a decisive role, although SALLE was a twice stacked sample extraction technique by organic solvent precipitation and salting-out. However, inspection of chromatograms obtained by UPLC-QTOF indicated that the use of SALLE (IPA/ $\mathrm{K}_{2} \mathrm{HPO}_{4}(4 \mathrm{M})$ ) allows the extraction of both hydrophilic and hydrophobic compounds from the plasma sample, because of the broad polar coverage of IPA [27]. Further removal of lipids is often required because the supernatant still contains many lipids that may cause severe matrix effects [22], which block the chromatographic column. It is also difficult to dissolve these lipids in aqueous chromatography mobile phase. In the present work, dichloromethane (DCM) was used to remove all the major lipid classes after completing the SALLE step. As shown in Fig. 1C, the chromatogram obtained using the SALLE along with DCM on a plasma sample was dramatically improved compared to that obtained by SALLE extraction. It is also clear from the chromatogram that there was no significant loss of IS during DCM extraction. 

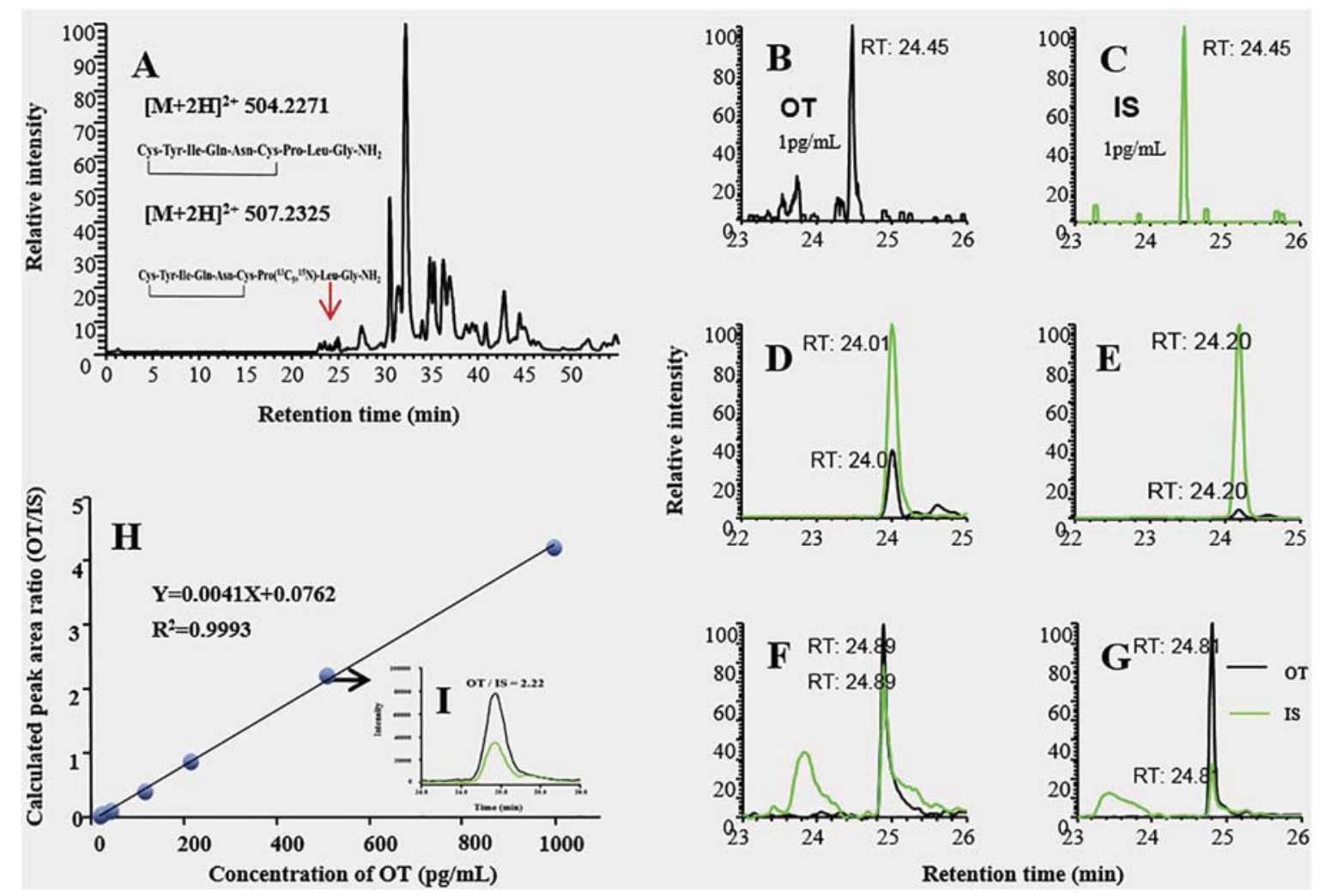

Fig. 2. The base peak chromatogram of human plasma obtained by nano-LC-MS in positive mode with the scaning range of 504.00-509.40 (A). The major precursor ions were $\mathrm{m} / \mathrm{z} 504.2271[\mathrm{M}+2 \mathrm{H}]^{2+}$ and $507.2325[\mathrm{M}+2 \mathrm{H}]^{2+}$ (doubly charged ion) for OT (B) and IS (C). The EIC of OT in two selected human plasmas with $200 \mathrm{pg} / \mathrm{m} \mathrm{L}$ of IS (D and E). The EIC of OT in two selected rat plasmas with $800 \mathrm{pg} / \mathrm{mL}$ of IS (F and G). The linear relationship between peak area ratio and various concentrations of oxytocin in $15 \%$ acetonitrile ( $0.1 \%$ formic acid, $0.05 \%$ human plasma) solution including regression equation and $\mathrm{R}^{2}$ value (H), and extracted ion chromatography (EIC) of OT at the concentration of $500 \mathrm{pg} / \mathrm{m} \mathrm{L}$, which yield with peak area ratio of 2.22 (I).

\subsection{Instant implement of SALLE}

We observed that the OT content was significantly reduced before sample pre-treatment under normal laboratory conditions. The commercial human plasma spiked with the OT standard was used to depict this binding phenomenon under different storage conditions. The degradation of OT was avoided by using a vacutainer with EDTA and enzyme inhibitors (DPP-IV, esterase and protease inhibitors). The $5 \% \mathrm{H}_{3} \mathrm{PO}_{4}$ strong acid solution was used to denature the proteins and to release OT. IS was added before the extraction step, and the peak area ratio between OT and IS was used to correct deviations that may occur during the use of SALLE and UPLC-MS.

As shown in Fig. 1D, the calculated peak area ratio of OT/IS reduced to $60 \%$ of the ' 0 ' time point after $4 \mathrm{~h}$, followed by reduction to $50 \%$ after $48 \mathrm{~h}$ at $23^{\circ}$ (red dots). On decreasing the storage temperature from $23^{\circ}$ to $18^{\circ}$, the loss of OT is still going on (blue squares). At $4^{\circ}$, the decrease of the OT/IS peak area ratio was obviously slowed down (Orange triangles). Presumably, this phenomenon is related to the strong protein binding of OT owing to disulfide bond exchange [19]. The similar phenomenon that low molecular weight biomarkers bind to albumin has been reported $[17,20]$. All these results confirmed that the binding of OT and protein occurs slowly and continuously after the plasma collection step. These OT-proteins adducts co-precipitated [28] during the pre-treatment step, thereby preventing the detection of OT. This may be the reason for the difference in the results of OT determination between labs and batches. Thus, we suggested that the storage time on the lab bench between plasma collection and the pre-treatment procedure must be taken into account for large sample analysis.
OT-protein binding can be effectively avoided by adding the SALLE reagent as soon as the plasma is taken out from the subjects (black dots in Fig. 1D). The OT content did not change dramatically for the next $48 \mathrm{~h}$ at $23^{\circ} \mathrm{C}$. The high concentration of salt and organic solvent in SALLE system can effectively precipitate protein into the intermediate layer prior to phase separation, which reduces the cross-reactivity of OT and protein. This means that the analysis and statistical separation of OT could be carried out using SALLE for a large number of clinical samples.

\subsection{Nano-LC-MS method validation}

Stable isotope-labelled internal standard-based quantification method using nano-LC-MS analysis was performed for the determination of endogenous OT. Fig. 2A shows the base peak chromatogram of human plasma obtained by nano-LC-MS in positive mode with the scaning range of 504.00-507.40, the major precursor ions were $504.2271[\mathrm{M}+2 \mathrm{H}]^{2+}$ and $507.2325[\mathrm{M}+2 \mathrm{H}]^{2+}$ (doubly charged ion) for OT (Fig. 2B) and IS (Fig. 2C), respectively. The LOQ concentration was $1.0 \mathrm{pg} / \mathrm{m} \mathrm{L}$ with the response of signal was 10 times that of noise. The sensitivity can be attributed to the ultra-high resolution of the Orbitrap spectrometer and capillary column. Fig. 2D-G shows the EIC of OT and IS in part of plasma sample. Fig. $2 \mathrm{H}$ shows the correlation between the calculated peak area ratio of OT/IS determined using nano-LC-MSLC-MS and the concentration of OT mentioned earlier, which indicate a good linear relationship $\left(R^{2}=0.9993\right)$. The Fig. 2I shows the superimposed extracted ion chromatography of OT at the concentration of $500 \mathrm{pg} / \mathrm{mL}$, which yield with peak area ratio of 2.22 .

The extraction efficiency was evaluated at three concentration levels ( $10 \mathrm{pg} / \mathrm{m} \mathrm{L}, 60 \mathrm{pg} / \mathrm{m} \mathrm{L}$, and $360 \mathrm{pg} / \mathrm{m} \mathrm{L}$ ) by comparing the peak 
Table 1

Precision, recovery, repeatability, matrix effect of oxytocin in plasma.

\begin{tabular}{|c|c|c|c|c|c|c|c|}
\hline \multirow{2}{*}{ Concentration $(\mathrm{pg} / \mathrm{mL})$} & \multirow[b]{2}{*}{ Intra-day } & \multicolumn{3}{|c|}{$\operatorname{Precision}(\mathrm{n}=5, \% \mathrm{RSD})$} & \multirow{2}{*}{ Absolute recovery (\%) } & \multirow{2}{*}{ Extract efficiency (\%) } & \multirow{2}{*}{ Matrix effect (\%) } \\
\hline & & Rt & Inter-day & Rt & & & \\
\hline 10 & 28.2 & 0.70 & 19.3 & 2.34 & 88.6 & 78.4 & 1.13 \\
\hline 60 & 13.4 & 0.90 & 13.2 & 1.16 & 81.6 & 85.1 & 0.96 \\
\hline 360 & 11.4 & 0.50 & 13.7 & 0.50 & 93.2 & 90.9 & 1.02 \\
\hline
\end{tabular}

Table 2

OT endogenous plasma concentrations in individual 12 humans and 6 rats.

\begin{tabular}{lllllll}
\hline Species & \multicolumn{6}{l}{ Endogenous OT level $(\mathrm{pg} / \mathrm{mL})$ in individual subjects } \\
\hline \multirow{4}{*}{ Human } & H1 & H2 & H3 & H4 & H5 & H6 \\
& 76 & 37 & 69 & 215 & 63 & 3 \\
& H7 & H8 & H9 & H10 & H11 & H12 \\
& 137 & 62 & 214 & 15 & 5 & 151 \\
\multirow{4}{*}{ Rat } & R1 & R2 & R3 & R4 & R5 & R6 \\
& 1253 & 2619 & 736 & 2111 & 145 & 354 \\
\hline
\end{tabular}

area ratios of OT spiked before and after extraction (Table1). In the pooled commercial plasma, the extract recovery and the absolute recovery varied from $81.6 \%$ up to $93.2 \%$ and from $78.4 \%$ to $90.9 \%$, respectively, which were corrected by adding IS prior to the SALLE step. ESI-MS is known to be sensitive to matrix effects, i.e. high salt content may alter the analyte signal [24]. As shown in Table 1, the matrix effects were $13 \%, 4 \%$ and $2 \%$, respectively, which once again suggests that SALLE is a sufficient method to remove salt from the plasma. The intra-day and inter-day precisions ranged from $11.43 \%$ to $28.24 \%$ and from $13.24 \%$ to $19.31 \%$, respectively. Sixty samples were successively injected without replacing the capillary column, again implying that SALLE can be used to extract clean abundant proteins and lipids from plasma.

\subsection{Measurement of intact oxytocin in human and rat Plasmas}

Applying the SALLE pre-treatment method and nano-LC-MS, we were able to detect ultra-trace level of intact oxytocin in plasma with good reliability. Therefore, the protocol was validated under physiological conditions. The individual plasmas were obtained from 12 volunteers and 6 rats. Identifications were based on the retention time matching the standards and spectra obtained with high-resolution MS. The stable isotope-labelled oxytocin was used as internal standards to compensate for the sample loss and instrumental variability. For each human plasma, the levels of intact OT ranged from 3 to $214 \mathrm{pg} / \mathrm{mL}$ (Table 2), the mean \pm SD was $87.2 \pm 75.2 \mathrm{pg} / \mathrm{mL}$, which were somewhat higher than that reported by Zhang [16]. On the one hand, the concentration of OT is reported to be closely related to the individuals and physiological states $[16,17]$. On the other hand, the proposed SALLE (IPA $/ \mathrm{K}_{2} \mathrm{HPO}_{4}$ $(4 \mathrm{M})$ ) is effective in removing large amounts of proteins, inorganic salts, and lipids to reduce matrix interference. Moreover, severe binding of OT to carrier proteins under in vitro conditions may also be responsible for the differences. The OT levels in the rat plasma were higher, varying from 145 to $2619 \mathrm{pg} / \mathrm{m} \mathrm{L}$, the mean \pm SD was $1203.0 \pm 988.9 \mathrm{pg} / \mathrm{m} \mathrm{L}$ than that of the human plasma.

\section{Conclusions}

A SALLE pre-treatment method coupled with nano-LC-MS was developed for measuring intact OT in human and rat plasmas. The cleanup step was not applied. The proposed SALLE (IPA $/ \mathrm{K}_{2} \mathrm{HPO}_{4}$ $(4 \mathrm{M})$ ) could efficiently remove disrupters, including abundant proteins, inorganic salt and lipids, which helps avoid the risk of blocking the capillary column and matrix effects. Continuous injection of 60 samples did not require replacement of the capillary column and showed good reproducibility. In addition, the plasma was immediately mixed with the SALLE reagent after thawing or collection to effectively prevent the binding of OT and protein. This new combination of SALLE and nano-LC-MS provided a very low LOQ of $1 \mathrm{pg} / \mathrm{mL}$ for intact OT in plasma. The hydrophilic organic solvent and salt composition can be tailored to a particular sample. Thus, the SALLE-nano-LC-MS approach may be an alternative method for the quantitative determination of other ultra-trace neuropeptides in plasma, which would help understand the role of neuropeptides in diverse biological functions.

\section{Conflict of interest}

The authors declare no competing financial interest.

\section{Acknowledgments}

We thank technical assistance from the mass spectrometry center of Dalian Institute of Chemical Physics. This work is supported by NSF81573830/81774154/21475128, the Projects MOST2015DFG42460 and CAS 100Talent Project.

\section{Appendix A. Supplementary data}

Supplementary material related to this article can be found, in the online version

\section{References}

[1] A.P. Borrow, N.M. Cameron, The role of oxytocin in mating and pregnancy, Horm. Behav. 61 (2012) 266-276.

[2] Z.R. Donaldson, L.J. Young, Oxytocin, vasopressin, and the neurogenetics of sociality, Sci. 322 (2008) 900-904.

[3] I.D. Neumann, Brain oxytocin: a key regulator of emotional and social behaviours in both females and males, J. Neuroendocrinol. 20 (2008) 858-865.

[4] D. Marazziti, M. Catena Dell'Osso, The role of oxytocin in neuropsychiatric disorders, Curr. Medical Chem. 15 (2008) 698-704.

[5] M. Althaus, Y. Groen, A.A. Wijers, H. Noltes, O. Tucha, P.J. Hoekstra, Oxytocin enhances orienting to social information in a selective group of high-functioning male adults with autism spectrum disorder, Neuropsychologia 79 (2015) 53-69.

[6] T. Sasaki, K. Hashimoto, Y. Oda, T. Ishima, T. Kurata, J. Takahashi, Y. Kamata, H. Kimura, T. Niitsu, H. Komatsu, M. Ishikawa, T. Hasegawa, A. Shiina, T. Hashimoto, N. Kanahara, T. Shiraishi, M. Iyo, Decreased levels of serum oxytocin in pediatric patients with attention deficit/hyperactivity disorder, Psychiat. Res. 228 (2015) 746-751.

[7] C. Modahl, L.A. Green, D. Fein, M. Morris, L. Waterhouse, C. Feinstein, H. Levin, Plasma oxytocin levels in autistic children, Biol. Psychatry 43 (1998) 270-277.

[8] M.E. McCullough, P.S. Churchland, A.J. Mendez, Problems with measuring peripheral oxytocin: can the data on oxytocin and human behavior be trusted? Neurosci. Biobehav. Rev, 37 (2013) 1485-1492.

[9] A. Szeto, P.M. McCabe, D.A. Nation, B.A. Tabak, M.A. Rossetti, M.E. McCullough, N. Schneiderman, A.J. Mendez, Evaluation of enzyme immunoassay and radioimmunoassay methods for the measurement of plasma oxytocin, Psychosom. Med. 73 (2011) 393-400.

[10] C. Péqueux, J.C. Hendrick, M.T. Hagelstein, V. Geenen, J.J. Legros, Novel plasma extraction procedure and development of a specific enzyme-immunoassay of oxytocin: application to clinical and biological investigations of small cell carcinoma of the lung, Scand. J. Clin. Lab. Invest. 61 (2001) 407-416.

[11] S. Haraya, K. Karasawa, Y. Sano, K. Ozawa, N. Kato, H. Arakawa, Development of a highly specific enzyme immunoassay for oxytocin and its use in plasma samples, Ann. Clin. Biochem. 54 (2017) 101-106. 
[12] B.A. Tabak, M.E. McCullough, A. Szeto, A.J. Mendez, P.M. McCabe, Oxytocin indexes relational distress following interpersonal harms in women, Psychoneuroendocrino 36 (2011) 115-122.

[13] D.R. Kommers, M. Broeren, P. Andriessen, S.G. Oei, L. Feijs, S. Bambang Oetomo, Pilot study demonstrates that salivary oxytocin can be measured unobtrusively in preterm infants, Acta Paediatr. 106 (2017) 34-42.

[14] H.R. Sobhi, B. Vatansever, A. Wortmann, E. Grouzmann, B. Rochat, Generic approach for the sensitive absolute quantification of large undigested peptides in plasma using a particular liquid chromatography-mass spectrometry setup, J. Chromatogr. A 1218 (2011) 8536-8543.

[15] E. Johnsen, S. Leknes, S.R. Wilson, E. Lundanes, Liquid chromatography-mass spectrometry platform for both small neurotransmitters and neuropeptides in blood, with automatic and robust solid phase extraction, Sci. Rep. 5 (2015) 9308.

[16] G. Zhang, Y. Zhang, D.M. Fast, Z. Lin, R. Steenwyk, Ultra sensitive quantitation of endogenous oxytocin in rat and human plasma using a two-dimensional liquid chromatography-tandem mass spectrometry assay, Anal. Biochem. 416 (2011) 45-52.

[17] Brandtzaeg, E. Johnsen, H. Roberg-Larsen, K.F. Seip, E.L. MacLean, L.R. Gesquiere, S. Leknes, E. Lundanes, S.R. Wilson, Proteomics tools reveal startlingly high amounts of oxytocin in plasma and serum, Sci. Rep. 6 (2016) 31693.

[18] https://pubchem.ncbi.nlm.nih.gov/compound/439302\#section=ComputedProperties.

[19] A.I. Mehta, S. Ross, M.S. Lowenthal, V. Fusaro, D.A. Fishman, E.F. Petricoin III, L.A. Liotta, Biomarker amplification by serum carrier protein binding, Dis. Mark. (2004) 1-10.

[20] C. Avanti, W.L.J. Hinrichs, A. Casini, A.C. Eissens, A. Van Dam, A. Kedrov, A.J.M Driessen, H.W. Frijlink, H.P. Permentier, The formation of oxytocin dimers is suppressed by the zinc-aspartate-oxytocin complex, J. Pharm. Sci. 102 (2013) 1734-1741.
[21] B.T. Roen, S.R. Sellevag, E. Lundanes, Quantification of nerve agent biomarkers in human serum and urine, Anal. Chem. 86 (2014) 11833-11840.

[22] M. Yoshida, A. Akane, M. Nishikawa, T. Watabiki, H. Tsuchihashi, Extraction of thiamylal in serum using hydrophilic acetonitrile with subzero-temperature and salting-out methods, Anal. Chem. 76 (2004) 4672-4675.

[23] F. Myasein, E. Kim, J. Zhang, H. Wu, T.A. El-Shourbagy, Rapid, simultaneous determination of lopinavir and ritonavir in human plasma by stacking protein precipitations and salting-out assisted liquid/liquid extraction, and ultrafast LC-MS/MS, Anal. Chim. Acta 651 (2009) 112-116.

[24] R.J. Raterink, P.W. Lindenburg, R.J. Vreeken, R. Ramautar, T. Hankemeier, Recent developments in sample-pretreatment techniques for mass spectrometry-based metabolomics, Trends Analyt. Chem. 61 (2014) 157-167.

[25] J. Zhang, R. Rodila, E. Gage, M. Hautman, L. Fan, L.L. King, H. Wu, T.A El-Shourbagy, High-throughput salting-out assisted liquid/liquid extraction with acetonitrile for the simultaneous determination of simvastatin and simvastatin acid in human plasma with liquid chromatography, Anal. Chim. Acta 661 (2010) 167-172.

[26] B.K. Matuszewski, M.L. Constanzer, C.M. Chavez-Eng, Strategies for the assessment of matrix effect in quantitative bioanalytical methods based on HPLC-MS/MS, Anal. Chem. 75 (2003) 3019-3030.

[27] A.L. Dawidowicz, E. Fornal, A. Fijalkowska, Problems in the analysis of propofol in blood when protein precipitation is used in sample preparation, Chromatographia 47 (1998) 523-528.

[28] M.H. Sarafian, M. Gaudin, M.R. Lewis, F.P. Martin, E. Holmes, J.K. Nicholson, M.E. Dumas, Objective set of criteria for optimization of sample preparation procedures for ultra-high throughput untargeted blood plasma lipid profiling by ultra-performance liquid chromatography-mass spectrometry, Anal. Chem. 86 (2014) 5766-5774. 